\title{
Correction to: HOXA11-AS promotes the migration and invasion of hepatocellular carcinoma cells by inhibiting miR-124 expression by binding to $\mathrm{EZH} 2$
}

Wen-long Zhang ${ }^{1} \cdot$ Ya-nan Zhao $^{1} \cdot$ Zhang-zhen Shi $^{1} \cdot$ Gui-ying Gu${ }^{1} \cdot$ Dan Cong ${ }^{1} \cdot$ Chen Wei $^{2} \cdot$ Yuan-song Bai $^{1} \mathbb{D}$

Published online: 17 September 2021

(c) Japan Human Cell Society 2021

\section{Correction to: Human Cell (2019) 32:504-514 \\ https://doi.org/10.1007/s13577-019-00269-x}

The original version of this article that appeared online was published with errors in Figs. 2, 3, 5 and 6. The revised figures are given in this correction.

The original article can be found online at https://doi.org/10.1007/ s13577-019-00269-x.

Yuan-song Bai

ysbai_cjuh@126.com

1 Department of Hematology and Oncology, China-Japan Union Hospital of Jilin University, No.126, Xiantai Street, Changchun 130033, Jilin, China

2 Zhongyuan Union Clinical Laboratory Co., Ltd, Beijing 100176, China 

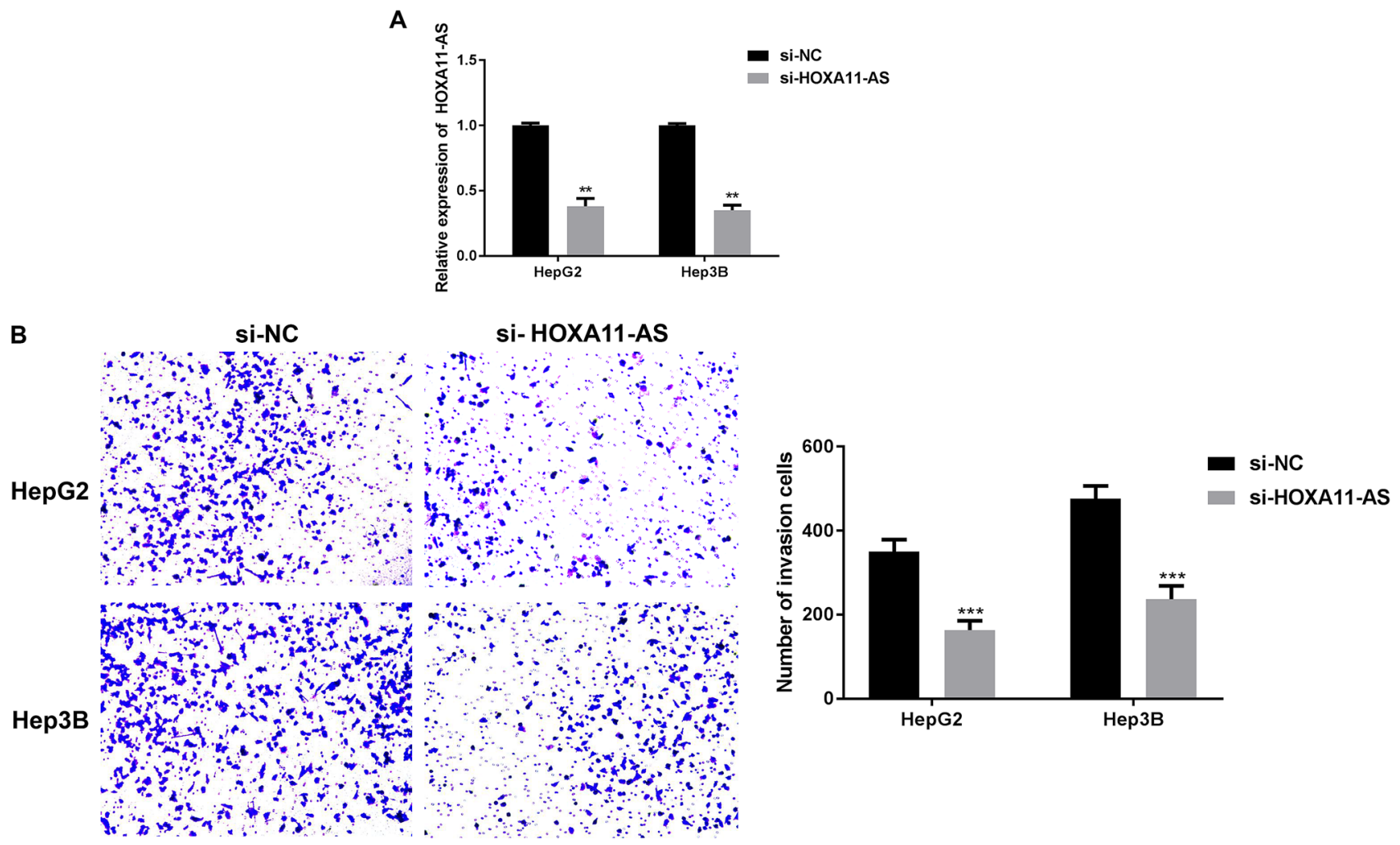

C

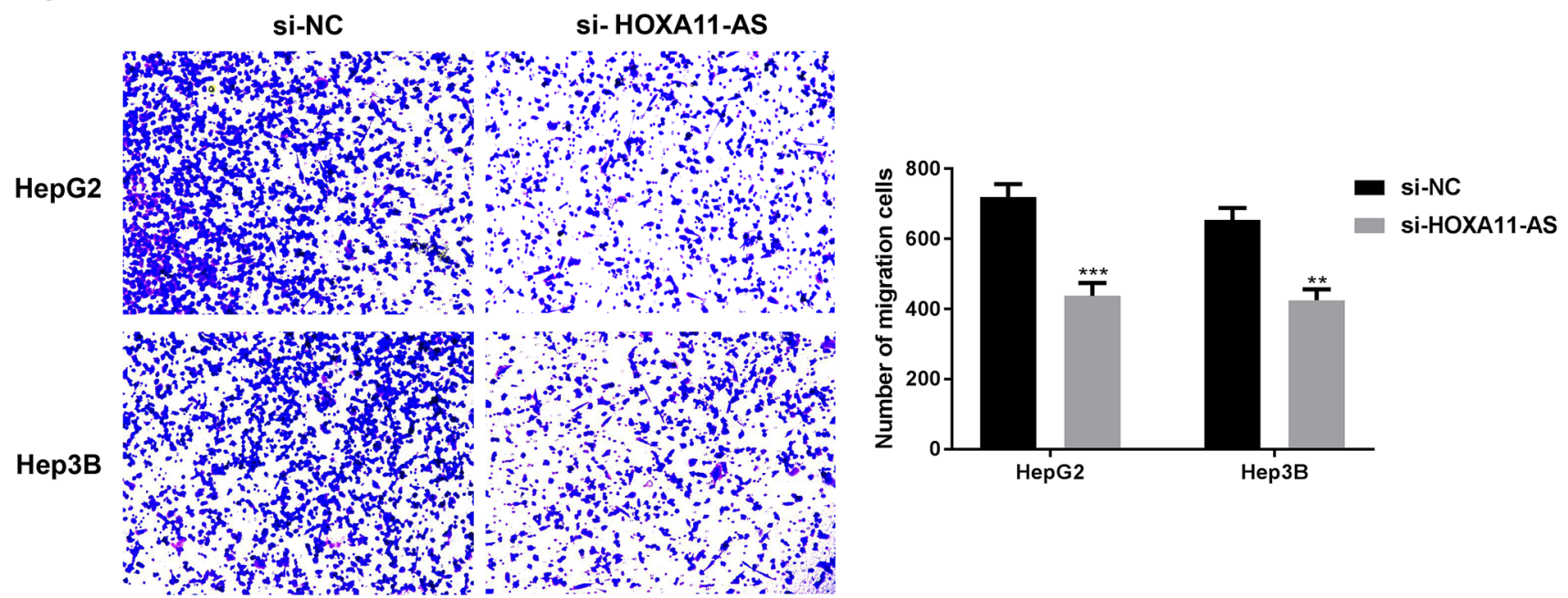

Fig. 2 Downregulation of HOXA11-AS inhibited HCC cell invasion and migration. a After transfection with si-HOXA11-AS, the relative expression of HOXA11-AS was much lower according to the qRT-
PCR. $* * P<0.01$. The ability of the HepG2 and Hep3B cells to invade (b) and migrate (c) was remarkably decreased after transfection with si-HOXA11-AS. $* * P<0.01$ 


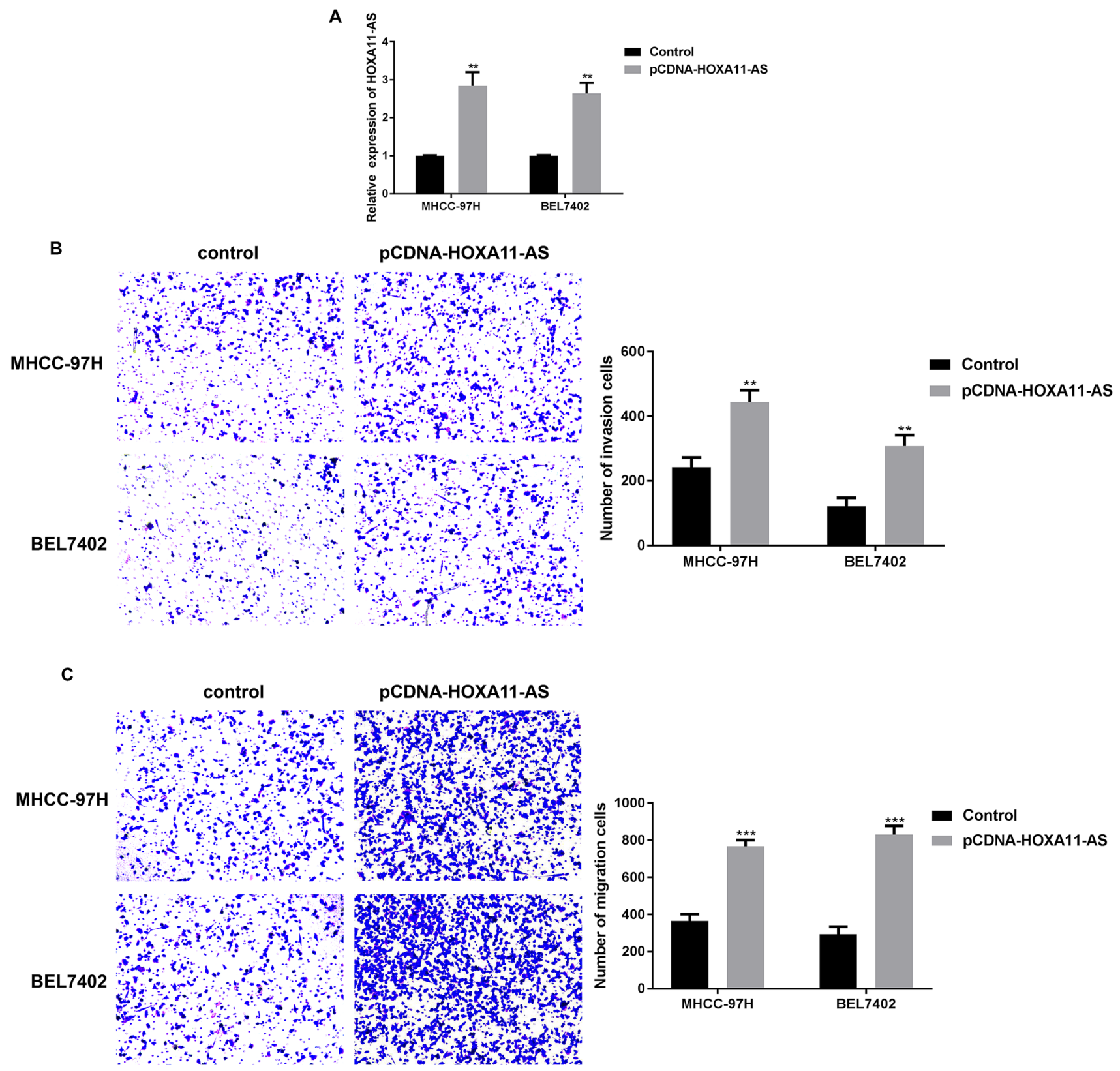

Fig. 3 Overexpression of HOXA11-AS promoted HCC cell invasion and migration. a Significantly increased HOXA11-AS levels were found in the MHCC-97H and BEL7402 cells after transfection with the pCDNA-HOXA11-AS vector. $* * P<0.01$. b A striking increase in the invasion of the MHCC-97H and BEL7402 cells was observed after pCDNA-HOXA11-AS transfection. $* P<0.05$. c A striking increase in the migration of the MHCC-97H and BEL7402 cells was observed after the pCDNA-HOXA11-AS transfection. $* * P<0.01$ 


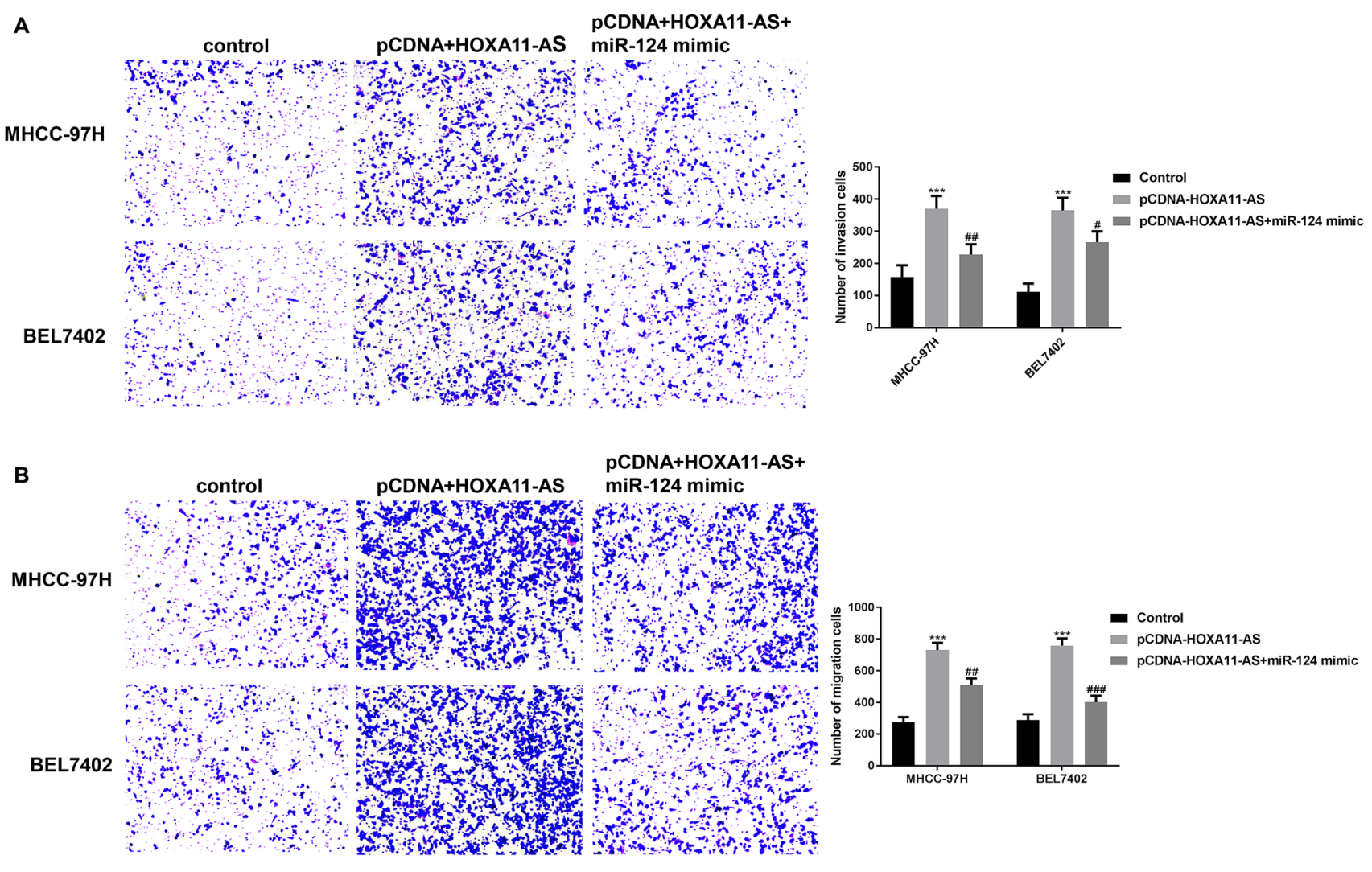

Fig. 5 Overexpression of miR-124 reversed the enhanced invasion and migration ability of the MHCC-97H and BEL7402 cells as induced by HOXA11-AS. a Overexpression of miR-124 reversed the enhanced invasion ability induced by HOXA11-AS. b Overexpres- sion of miR-124 reversed the enhanced migration ability induced by HOXA11-AS. $* * P<0.01$ : pCDNA-HOXA11-AS versus control; ${ }^{\#} P<0.001$ : pCDNA-HOXA11-AS + miR-124 mimic versus pCDNA-HOXA11-AS 
A

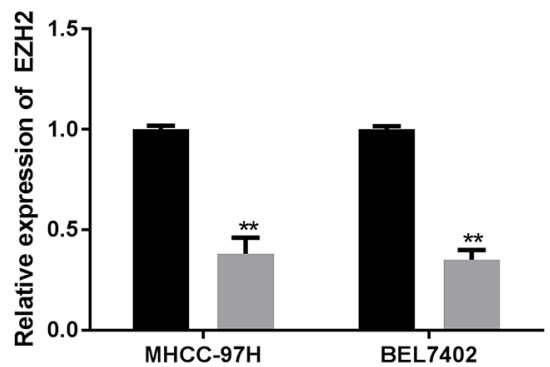

si-EZH2

si-NC MHCC-97H BEL7402
si-NC

si-EZH2

EZH2

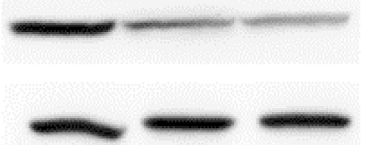

B

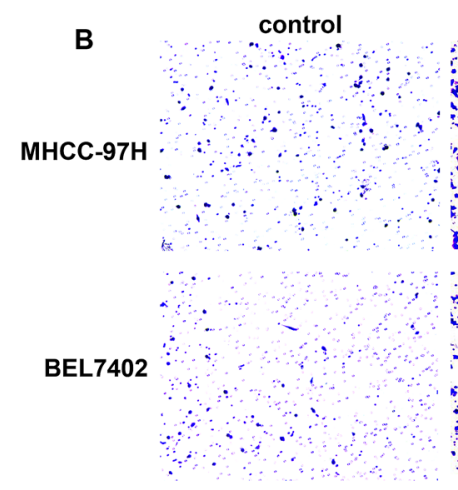
control

PCDNA+HOXA11-AS+

pCDNA+HOXA11-AS Si-EZH2

$\beta$-actin
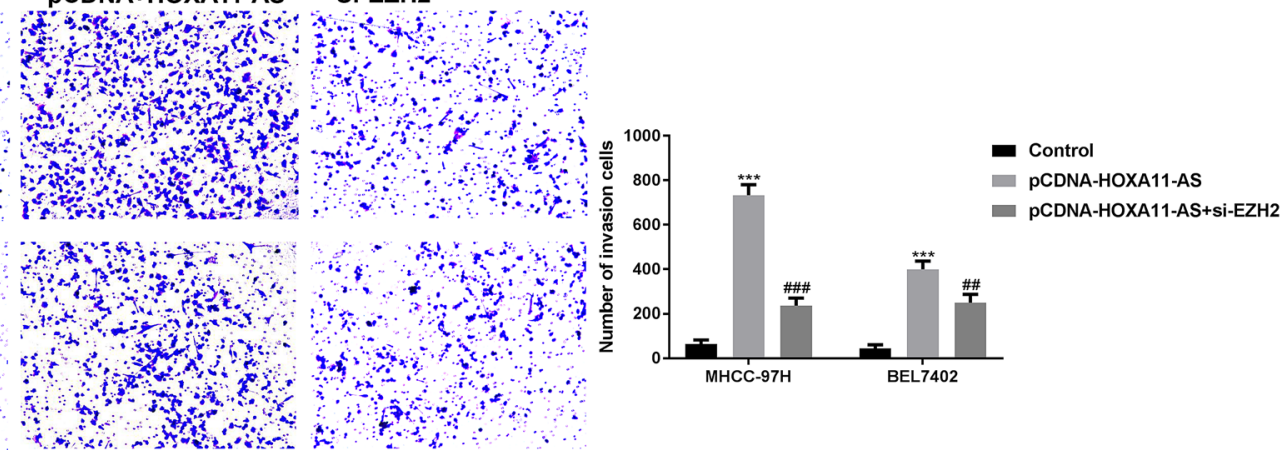

C

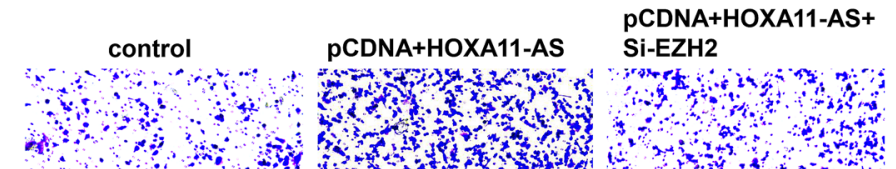

MHCC-97H

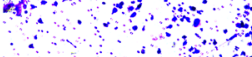
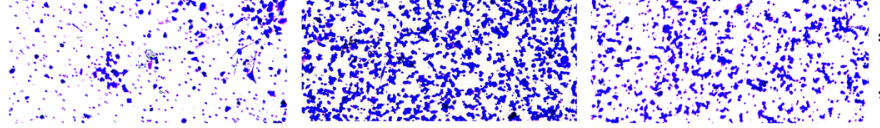

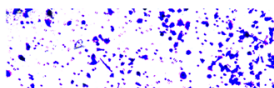

BEL7402
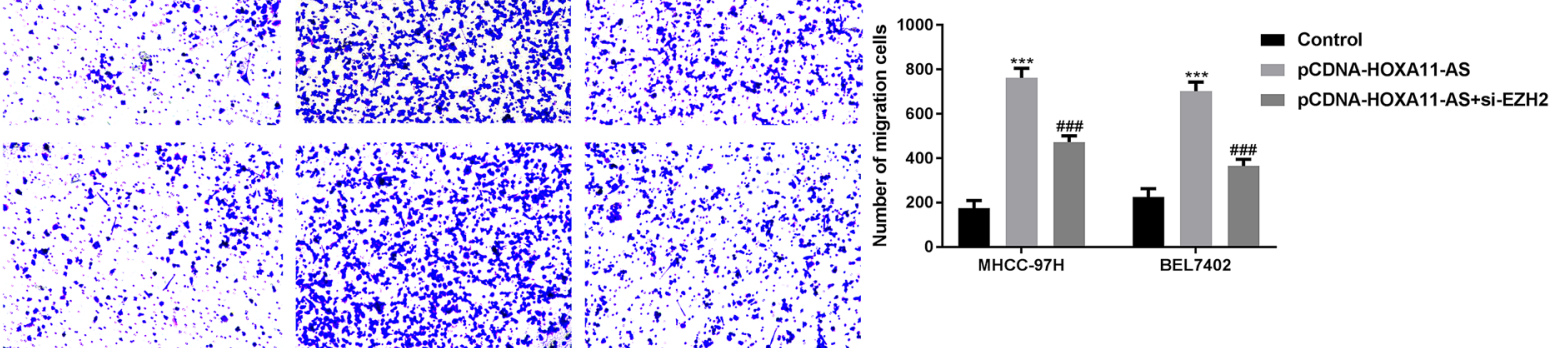

Fig. 6 Silencing the expression of EZH2 reverses the cell invasion and migration effects induced by HOXA11-AS. a EZH2 expression was significantly decreased in the MHCC-97H and BEL7402 cells after transfection with EZH2 siRNA. $* * P<0.01$. b EZH2 silencing reversed the HCC cell invasion ability induced by HOXA11-AS.
cEZH2 knockdown reversed the HCC cell migration ability induced by HOXA11-AS. $* * P<0.01$ : pCDNA-HOXA11-AS versus control; ${ }^{\# \#} P<0.001$ : pCDNA-HOXA11-AS + si-EZH2 versus pCDNAHOXA11-AS

Publisher's Note Springer Nature remains neutral with regard to jurisdictional claims in published maps and institutional affiliations. 